\title{
Effects of static electricity and fabrication parameters on PVDF film properties
}

\author{
MOHAMMAD HOSSEIN GHAJAR ${ }^{1,2, *} \mathbb{D}$, MAHMOUD MOUSAVI MASHHADI ${ }^{\mathbf{1}}$, \\ MEHRDAD IRANNEJAD ${ }^{2}$, MUSTAFA YAVUZ ${ }^{2}$ and EIHAB ABDEL-RAHMAN ${ }^{2}$ \\ ${ }^{1}$ Department of Mechanical Engineering, University of Tehran, 1417614418 Tehran, Iran \\ ${ }^{2}$ Waterloo Institute for Nanotechnology, University of Waterloo, Waterloo N2L 3G1, Canada \\ *Author for correspondence (m.ghajar@ut.ac.ir)
}

MS received 6 May 2017; accepted 1 September 2017; published online 28 March 2018

\begin{abstract}
Degree of crystallinity and $\beta$-phase fraction are important factors in determining electroactive polymers performance. In the present work, effects of intrinsic static electricity, substrate type, PVDF solution concentration and drying temperature on $\beta$-phase fraction and degree of crystallinity in fabricated PVDF films were studied using XRD, Raman spectroscopy and FTIR techniques. In particular, this paper investigates the influence of static electricity on PVDF film properties for the first time. The results show that discharging static electricity from the PVDF solution is highly effective in reducing the amount of residual solvent. It was found that a lower amount of residual solvent and higher drying temperature resulted in a higher degree of crystallinity. Further, a high fraction of $\beta$ phase was observed in all PVDF films due to more polar solvent effect that it was hardly affected by other parameters such as static electricity.
\end{abstract}

Keywords. Polyvinylidene fluoride; electrostatic field; substrate effect; degree of crystallinity; fraction of $\beta$ phase.

\section{Introduction}

Electroactive polymers (EAPs) are smart polymers that change their size or shape in the presence of an electric field. Polyvinylidene fluoride (PVDF) and its copolymers possess the best electroactive properties among all polymers [1] and hence, were widely used in sensors, actuators and energy harvesters [2-4]. In addition, they have excellent mechanical and chemical stability, processability and low-mechanical and acoustic impedance [5].

PVDF is a semicrystalline polymer observed in four different crystalline phases $\alpha, \beta, \gamma$ and $\delta$. The quantity of each crystalline phase depends on the particular preparation conditions, such as solution concentration and drying temperature [6]. Chain conformation for $\alpha, \beta$ and $\gamma$ phases are TGTG', TTTT (planar zigzag) and $\mathrm{T}^{\mathrm{G}} \mathrm{GT} 3 \mathrm{G}^{\prime}$, respectively, where T and $\mathrm{G}$ stand for trans and gauche linkages, respectively $[7,8]$.

A PVDF monomer unit contains two hydrogen and two fluorine atoms connected to the carbon backbone. The electronegativity of fluorine is much higher than the electronegativity of hydrogen and carbon elements, which results in a permanent electric dipole moment in PVDF chain, which is perpendicular to the chain axis [9]. This polarity in PVDF chains leads to piezo- pyro- and ferro-electric properties. The highest amount of electric dipole moment occurs in the planar zigzag $\beta$ formation [10].

There are many ways to fabricate PVDF films in a specific crystalline phase. For example, by using solution-casting method, different phases can be achieved by adjusting the drying temperature, type of solvent, solution concentration, ambient conditions, solvent evaporation rate and mechanical condition (e.g. stretching PVDF sample in one or two directions) [7,11-15]. Also, poling, spin coating, electro-spinning and use of additives like clay result in the polar $\beta$ phase of PVDF [16]. For example, Dhakras et al [16] prepared PVDF nanofibres using electrospinning and they significantly enhanced its piezoelectric properties by addition of a hydrated salt, $\mathrm{NiCl}_{2} \cdot 6 \mathrm{H}_{2} \mathrm{O}$.

In recent years, the effects of solution concentration and drying temperature on the type of the phase formation in PVDF films were studied extensively $[1,6,10,17,18]$. This paper studies the effects of intrinsic static electricity and substrate type, in addition to solution concentration and drying temperature, on the degree of crystallinity and phase formation in PVDF films.

\section{Experimental}

A series of PVDF films were fabricated by drop-casting $1 \mathrm{ml}$ of PVDF solution on four different types of flat substrates. Three PVDF solutions were prepared by dissolving 5, 10 and $20 \%$ (by weight) PVDF powder [19] in dimethylacetamide (DMAc) solvent [20]. The solution is drop-cast on Teflon, pyrex, stainless steel (SS) and grounded stainless steel (GSS) substrates. Finally, all the samples are dried using a hot-plate at a constant temperature as detailed in table 1. 
Table 1. Fabrication parameters of PVDF films.

\begin{tabular}{lccc}
\hline Sample & PVDF concentration $(w t \%)$ & Substrate & Drying temperature $\left({ }^{\circ} \mathrm{C}\right)$ \\
\hline 10Teflon42 & 10 & Teflon & 42 \\
10 Pyrex42 & 10 & Pyrex & 42 \\
10 SS42 & 10 & Stainless steel & 42 \\
10 GSS42 & 10 & Grounded stainless steel & 42 \\
10GSS30 & 10 & Grounded stainless steel & 30 \\
20GSS60 & 20 & Grounded stainless steel & 60 \\
20GSS70 & 20 & Grounded stainless steel & 70 \\
5GSS120 & 5 & Grounded stainless steel & 120 \\
\hline
\end{tabular}

Teflon and Pyrex are common substrates in the fabrication of PVDF films by drop-casting method [6,21]. In addition to these substrates, a stainless steel substrate was used because it is conductive and at the same time it has perfect chemical resistance. Therefore, it can be grounded and remove static electrical charges out from the system. To determine the role of natural static electricity, a stainless steel substrate was used, which was not grounded and the electrical charges would be remained in the system. Choosing these substrates provides a wide range of surface morphology and materials type from crystalline solid to amorphous solid and polymer that can fabricate using various PVDF film formations.

A naturally induced electrostatic field was observed in the PVDF powder before mixing with DMAc solvent when it was absorbed into the container wall. The induced charges applied an electric field to the PVDF solution during the drying process and affected crystallization. Connecting the substrate to ground resulted in discharging the induced static electricity and eliminating the electrostatic field. Grounded stainless steel (GSS) in table 1 refers to the stainless steel substrates treated in this manner. The effects of electrostatic field and substrate material on PVDF films properties were studied in the first four specimens, namely 10 Teflon 42 , 10Pyrex42, 10SS42 and 10GSS42. However, the effects of solution concentration and drying temperature on the PVDF films properties were investigated in the last four specimens, namely 10GSS30, 20GSS60, 20GSS70 and 5GSS120.

The structural characterization of the PVDF films was carried out using X-ray diffractogram technique (PANalytical X'pert powder XRD), Fourier transform infrared spectroscopy (Bruker Tensor FTIR spectrometer) and Raman spectroscopy (Renishaw Raman-Microramn spectrometer). The Raman spectra were recorded in the range of 200$1600 \mathrm{~cm}^{-1}$ using excitation wavelength of $632.8 \mathrm{~nm}$ and power density of $10 \mathrm{~mW} \mathrm{~cm}^{-2}$. The thickness of all the samples was measured by a micrometer caliper.

\section{Results and discussion}

The influence of electrostatic field, substrate, the solution concentration and drying temperature on residual solvent, degree

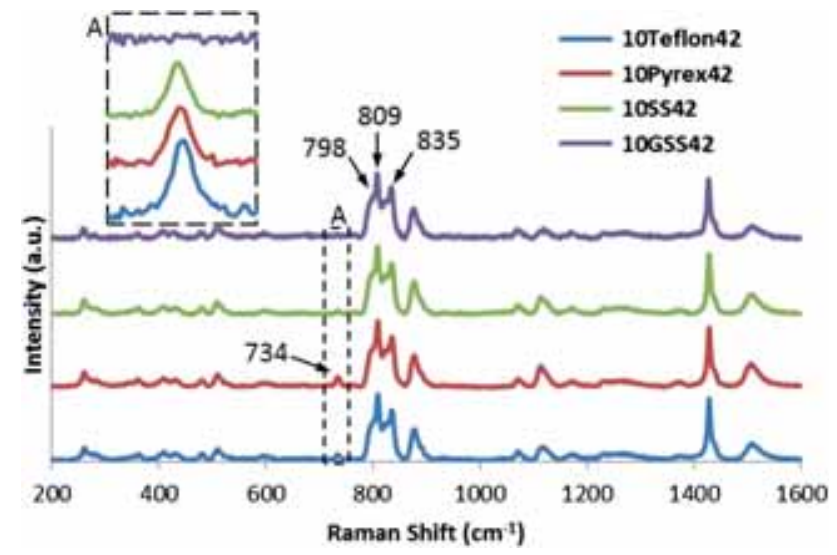

Figure 1. The recorded Raman spectra of PVDF films cast on different substrates. The excitation wavelength was fixed at $632.8 \mathrm{~nm}$ and power density of $10 \mathrm{~mW} \mathrm{~cm}{ }^{-2}$. The inset shows higher magnification of Raman spectra at around $734 \mathrm{~cm}^{-1}$.

of crystallinity and the amount of $\beta$-phase fraction of the fabricated PVDF films were systematically studied.

\subsection{Residual DMAc}

Raman spectra of 10Teflon42, 10Pyrex42, 10SS42 and 10GSS42 are compared in figure 1. The details of the DMAc solvent-related Raman features [22] are shown in figure 1 inset. As can be seen from this figure, the DMAc vibration peak was recorded at $734 \mathrm{~cm}^{-1}$ for the Teflon, Pyrex and stainless steel substrates. However, this peak was completely absent for the grounded stainless steel substrate. The presence of DMAc residue in the Teflon, Pyrex and stainless steel substrate samples could be attributable to the formation of a weak electrostatic bond at the interface, which resulted in trapping solvent molecules inside the polymer chains network and prevented their evaporation. The intrinsic electrostatic field can influence the PVDF crystallization in two ways; firstly, by itself, and secondly, by the residual solvent. It is known that there is a permanent dipole in PVDF monomers and DMAc molecules. Therefore, PVDF chains interact with both electrostatic field and DMAc solvent. Theoretically, electrostatic 
field could mostly affect PVDF chains to form in polar $\beta$ phase. On the other hand, polar solvent molecules act as a $\beta$ nucleus and thus results in PVDF films that were formed in extremely high $\beta$-phase content. At the same time, existence of polar solvent disturbs the growth of crystalline domain. Hence, it can be concluded that electrostatic field creates residual polar solvent, which decreases the amount of crystalline area by disturbing spherulite growth.

\subsection{Degree of crystallinity}

The ratio of crystalline content to amorphous content is known as polymer crystallinity [15]. It determines polymer properties, such as hardness, stiffness, melting point and electroactive properties [18]. The degree of crystallinity of the PVDF films can be calculated with the help of XRD pattern. By deconvoluting the PVDF XRD pattern, the crystalline phases are given by sharp peaks, while the amorphous phases are given by broader peaks. The degree of crystallinity is calculated by dividing the area of crystalline peaks by the summation of the crystalline and non-crystalline peak areas
[15]. The XRD patterns of all fabricated PVDF films are compared in figures 2 and 3 . In both the figures, the crystalline and amorphous areas are shown as black and gray peaks, respectively.

The calculated degree of crystallinity of PVDF films fabricated from different PVDF concentrations at different drying temperatures on different substrates are presented in figure 4. In first four films fabricated from $10 \mathrm{wt} \%$ PVDF solution at $42^{\circ} \mathrm{C}$, all fabrication parameters were the same except for the substrate. As expected, the degree of crystallinity of PVDF films is affected by the substrate material as shown in figure 4 . There is an interaction between PVDF chains and substrate surface. Different substrates result in different nucleations and crystallinities of PVDF chains at the interface due to surface energy, roughness and lattice matching of polymer crystal and substrate [23]. PVDF chains near the substrate try to form in the phase that its crystal parameters are closer to the substrate crystal. Surface energy by definition is the increase in free energy that accompanies an increase in surface area [24]. A specific surface energy exists between PVDF solution and each substrate that results in interactions between them, which affect the
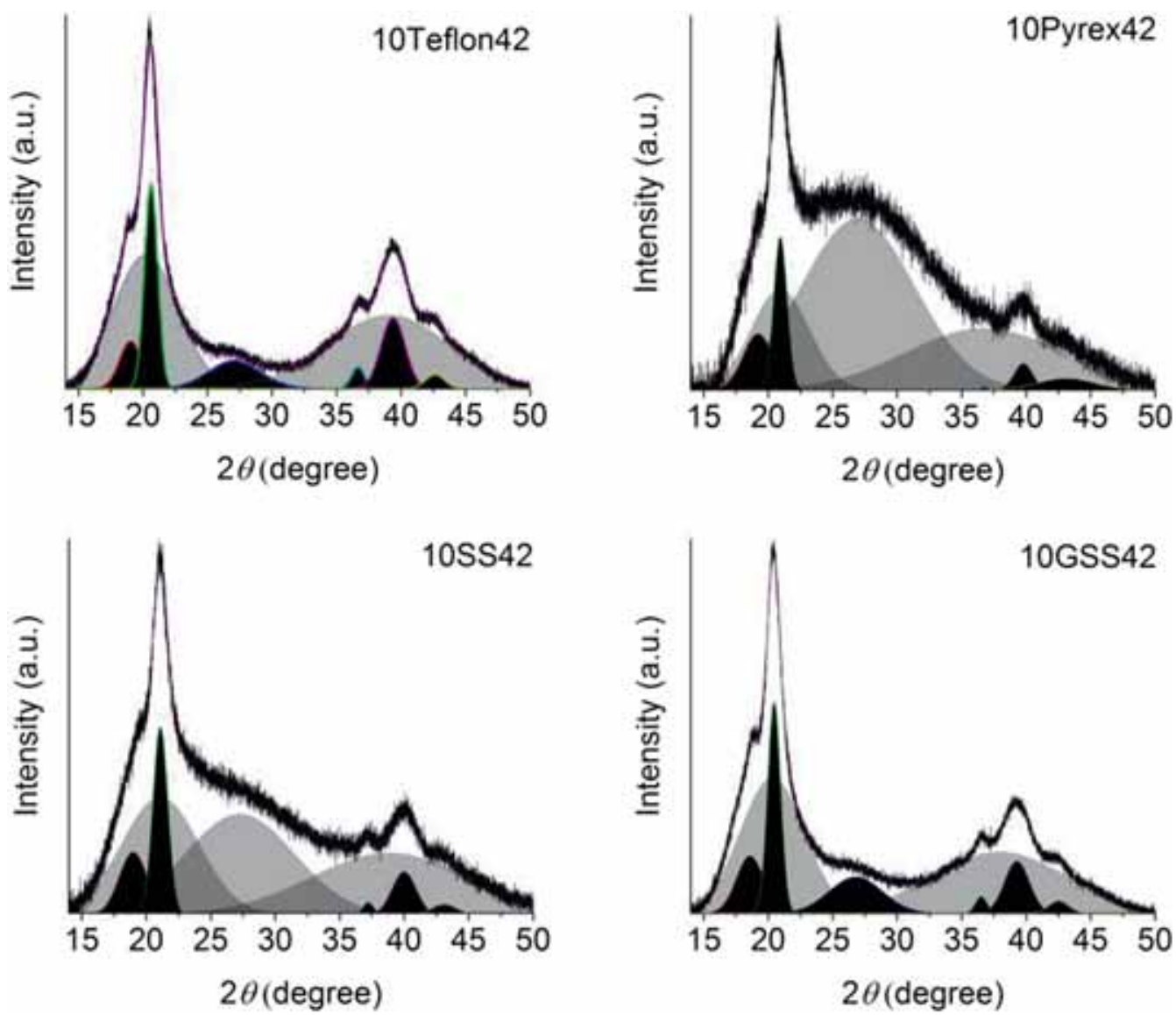

Figure 2. The recorded XRD pattern of PVDF films cast on different substrates. The deconvoluted black and grey peaks show the crystalline and amorphous phases, respectively. All PVDF films were fabricated using $10 \mathrm{wt} \%$ PVDF and drying temperature of $42^{\circ} \mathrm{C}$. 

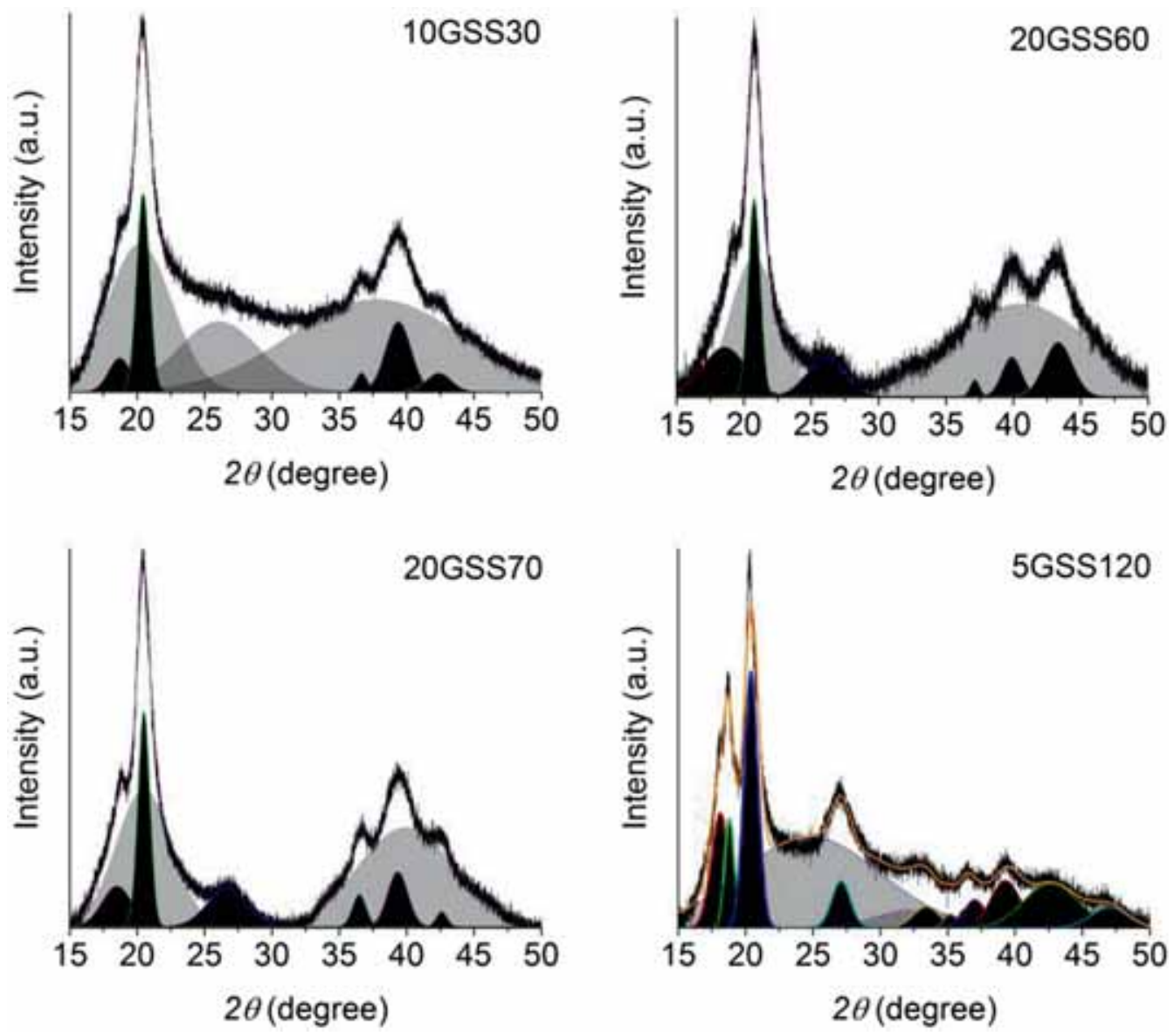

Figure 3. The recorded XRD patterns of PVDF films are formed at different solution concentrations and drying temperatures. The deconvoluted black and grey peaks show the crystalline and amorphous phases, respectively. In all the samples, grounded stainless steel was used as a substrate.

\section{Degree of Crystallinity (\%)}

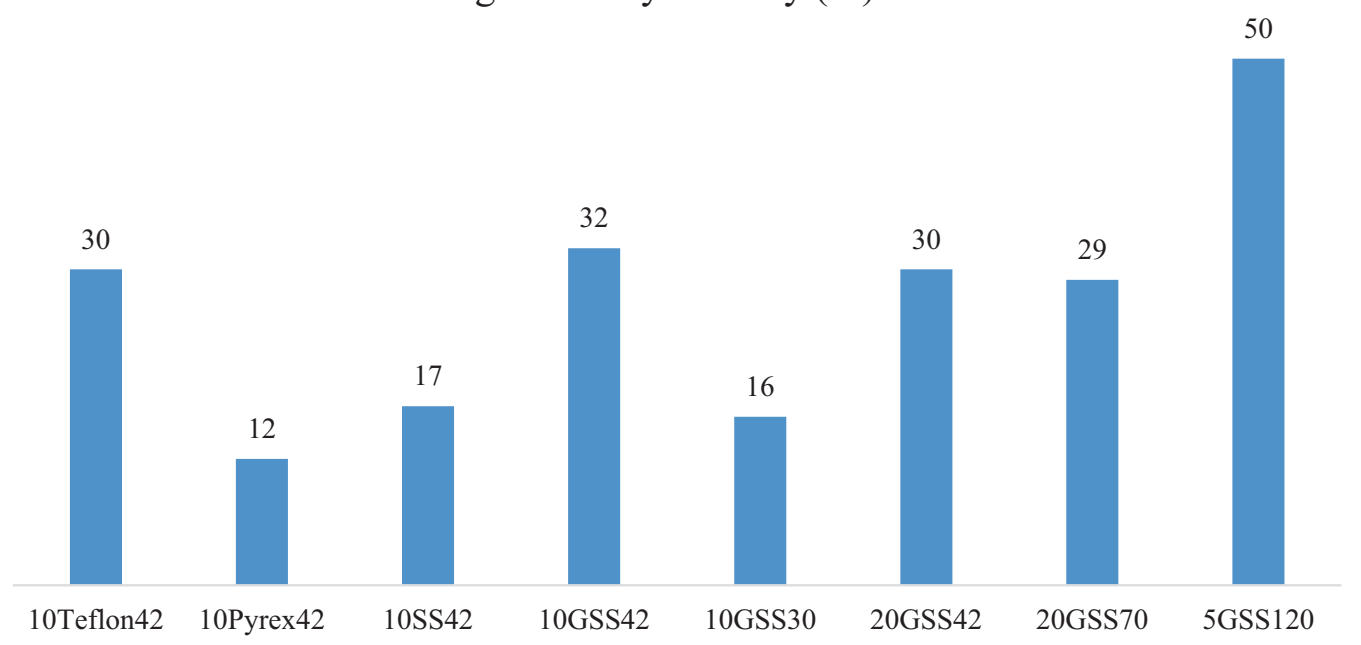

Figure 4. Calculated degree of crystallinity of PVDF films formed using different drying temperatures, different concentrations of PVDF and different substrates. 
crystallization process. Furthermore, roughness can influence the forces applied to PVDF chains via Wenzel's model or trapped air. Comparison of PVDF films formed on stainless steel and grounded stainless steel substrates (10SS42 and 10GSS42) show that the degree of crystallinity was affected by electrostatic field, or in other words, it was affected by solvent residue. It is known that DMAc molecules and PVDF monomer units are polar. Thus, DMAc molecules interact with PVDF chains due to the electrostatic forces between them. In consequence, the presence of DMAc molecules disturbs the crystallization mechanism of PVDF by confining spherulite growth. A similar observation was reported for PVDF incorporating ionic liquid, in which PVDF chains interact with ions via electrostatic forces [25]. From figure 4, it is evident that the degree of crystallinity of PVDF film formed on Teflon was almost the same as that on GSS substrate, despite the presence of the polar solvent in 10Teflon42. This increase in degree of crystallinity for Teflon could be attributed to the effect of the lower surface energy and higher roughness of the Teflon substrate in crystallization process [26]. These results suggest the need for use of a conducting substrate that can be grounded with low surface energy and high roughness to have a high degree of crystallinity for better mechanical properties. In contrast, a vice versa circumstance is needed for increasing amorphous phase to obtain high ion mobility. Designing a proper EAP remains as an engineering challenge in which the degree of crystallinity is required to be optimized for the best performance.

Recently, Chinaglia et al [6] and Cardoso et al [10] reported some methods for fabrication of PVDF films in $\alpha$ and $\beta$ phases. These methods were utilized in this research to produce PVDF films to study their crystalline properties for the purpose of fabrication of EAPs. The calculated degree of crystallinity of formed PVDF films from Chinaglia et al [6] and Cardoso et al [10] methods by using GSS substrate are compared in last four samples of figure 4 . It is clear that by increasing drying temperature and evaporation rate, polymer crystallinity was increased as expected. DMAc molecules act as a nucleation agent and thus, it increases the crystallinity. In contrast, as it mentioned before, DMAc molecules confine growth of spherulite and therefore, it decreases crystallinity. It is known that higher drying temperature results in higher crystallization kinetics and larger evaporation rate. Therefore, faster spherulites growth is expected with less growth confinement caused by DMAc solvent. In other words, DMAc molecules mainly act as nucleation agents rather than confining crystallization at higher temperature. Here, we did not consider the effect of solution concentration on degree of crystallinity and it is neglected due to evaporation rate slightly changes when solution concentration changes at low temperature, and secondly, the effect of temperature change is significantly larger than the effect of solution concentration change [6]. The results indicate that high drying temperature is recommended to achieve a higher mechanical properties, and low drying temperature to have higher ion mobility.

\subsection{Phase characterization}

In general, PVDF could be seen in four different crystalline phases. Most dominant phases are $\alpha$ and $\beta$. Fraction of $\beta$ phase, $F(\beta)$, could be calculated using FTIR analysis and equation (1) [27];

$$
F(\beta)=\frac{A_{\beta}}{1.26 A_{\alpha}+A_{\beta}},
$$

where $A_{\alpha}$ and $A_{\beta}$ are the intensity of absorbance correspond to absorption bands at 763 and $840 \mathrm{~cm}^{-1}$, respectively. The FTIR spectrum of fabricated PVDF films using different substrates, $10 \mathrm{wt} \%$ of PVDF solution and drying temperature of $42^{\circ} \mathrm{C}$ are compared in figure 5. The FTIR spectrum of fabricated PVDF on the grounded stainless steel substrate, different PVDF concentrations and different drying temperature are compared in figure 6. From these two figures, it is clear that $\beta$ phase was the most dominant phase in all the fabricated films. From figure 6 inset, it can also be seen that $A_{\alpha}$ and $A_{\beta}$ are slightly different among 10GSS30, 20GSS60, 20GSS70 and 5GSS120 films qualitatively indicating that the highest and lowest $\beta$ fractions are obtained in 20GSS60 and 5GSS120 PVDF films, respectively.

Figure 7 shows the calculated $F(\beta)$ of the formed PVDF films using different substrates, different PVDF concentrations and different drying temperatures. $F(\beta)$ was calculated using equation (1) and the intensity of absorption bands at wavenumbers of 763 and $839 \mathrm{~cm}^{-1}$. Figure 8 shows deconvoluted FTIR spectrum of 5GSS120 PVDF film as an example, in which $A_{\alpha}$ and $A_{\beta}$, the intensity of the absorption bands at wavenumbers of 763 and $839 \mathrm{~cm}^{-1}$, are investigated by black line. As can be seen from figure 7, $F(\beta)$ of all fabricated PVDF films were calculated in the range of $82-96 \%$. Because of using drop-casting method and polar solvent, $F(\beta)$ of all the samples were relatively high. Indeed, DMAc molecules act as $\beta$ nucleation agents because of its polarity and force PVDF chains to form and grow in $\beta$-phase

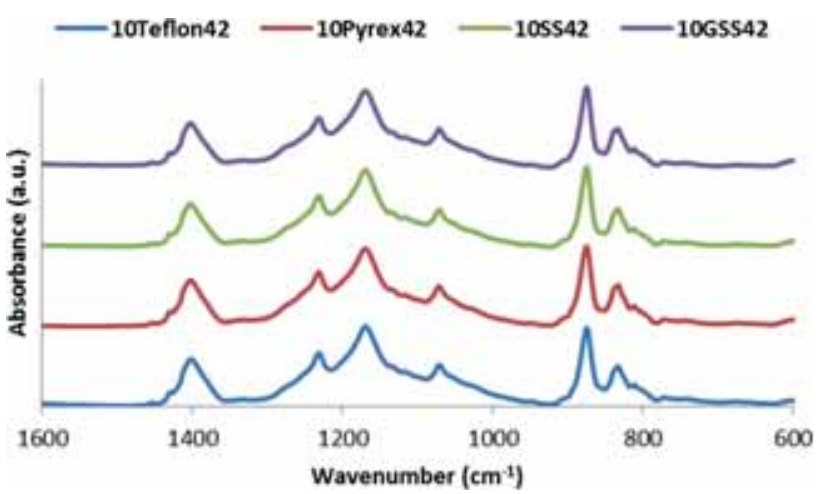

Figure 5. The recorded FTIR spectrum of fabricated PVDF films using different substrates, $10 \mathrm{wt} \% \mathrm{PVDF}$ and drying temperature of $42^{\circ} \mathrm{C}$. 
formation. $F(\beta)$ of 10Teflon42, 10Pyrex42, 10SS42 and $10 \mathrm{GSS} 42$ were calculated in the range of $85-89 \%$, which is approximately constant, and indicating that the effects of neither electrostatic field nor substrate on $F(\beta)$ could not be detected at such high values. By comparing 10GSS30, 20GSS60, 20GSS70 and 5GSS120 PVDF films, the maximum $F(\beta)$ was obtained in the film with $20 \mathrm{wt} \%$ of PVDF solution and drying temperature of $60^{\circ} \mathrm{C}$ (20GSS60). While the lowest one was acquired in the fabricated film from 5 wt $\%$ of PVDF solution and drying temperature of $120^{\circ} \mathrm{C}$ (5GSS120). In consequence, casting the denser solution at

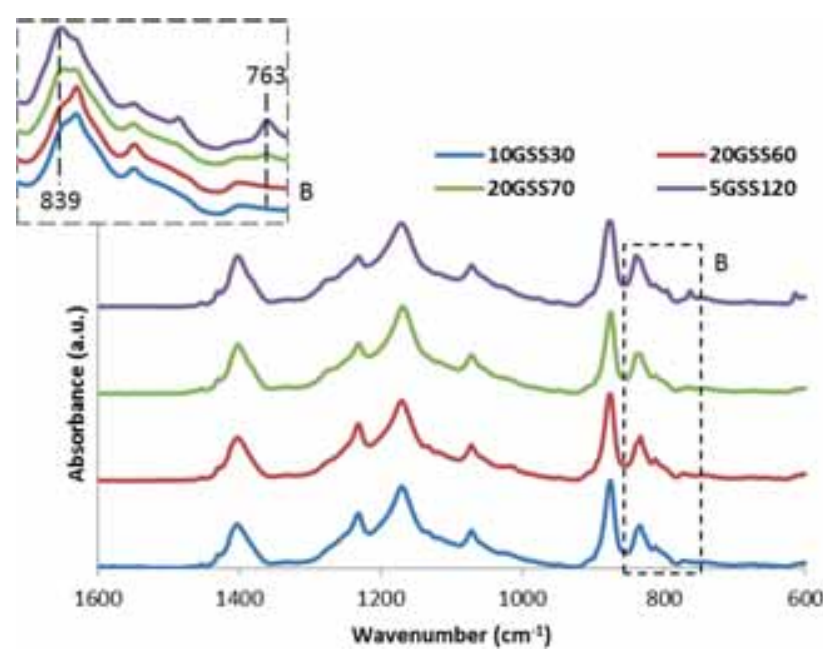

Figure 6. The recorded FTIR spectrum of formed PVDF films on the grounded stainless steel substrate was from different concentrations of PVDF and different drying temperatures in the range of $30-120^{\circ} \mathrm{C}$. The inset shows the higher magnification of FTIR spectra in the range of $760-840 \mathrm{~cm}^{-1}$. lower drying temperature resulted in a high $F(\beta)$. This could be attributed to the lower evaporation rate and hence, sufficient time for $\beta$-phase formation, as this phase is more stable than $\alpha$ phase [6]. Therefore, these results suggest that a high PVDF concentration in DMAc solvent and a drying temperature of $60^{\circ} \mathrm{C}$ for fabrication of PVDF films with a high fraction of $\beta$ phase.

Raman spectroscopy was also carried out in addition to FTIR spectroscopy to study the produced phases in the fabricated PVDF films. The recorded Raman spectrum of the PVDF films formed using different concentrations of the PVDF in the range of 5-20 wt\% and different drying temperatures in the range of $30-120^{\circ} \mathrm{C}$ are shown in figures 1 and 9 . As it is clear from these figures, $\beta$ phase was the main crystallinity phase in the recorded Raman spectrum [28]. It was also found that the recorded intensity at wavenumber of $798 \mathrm{~cm}^{-1}$ in 5GSS120 PVDF film (purple curve) is larger than that one using other concentrations and drying temperatures as shown in figure 9. As it can be seen, increases in the recorded intensity at the wavenumber of $798 \mathrm{~cm}^{-1}$ resulted in a reduction in Raman peaks intensity at wavenumbers of 809 and $835 \mathrm{~cm}^{-1}$ in 5GSS120 PVDF film. This behaviour in the recorded Raman spectrum could be contributed to the formation of lower $\beta$-phase and larger $\alpha$ phase in 5GSS120 PVDF film compared to PVDF films formed using other parameters as summarized in table 1 . The Raman spectra confirm the FTIR analysis presented in figure 7 . The recorded Raman peaks at wavenumbers of 283,608 and $798 \mathrm{~cm}^{-1}$ is assigned to $\alpha$ phase. While, the related $\beta$-phase peaks are recorded at wavenumbers of 262,835 and $1429 \mathrm{~cm}^{-1}$ [28]. The recorded peak at wavenumber of $809 \mathrm{~cm}^{-1}$ can be attributed to $\gamma$ phase formation in the PVDF films [29]. By comparing the recorded Raman spectrum of the PVDF films formed at drying temperature of $120^{\circ} \mathrm{C}$ (5GSS120) with those formed

\section{Fraction of $\beta$ Phase (\%)}

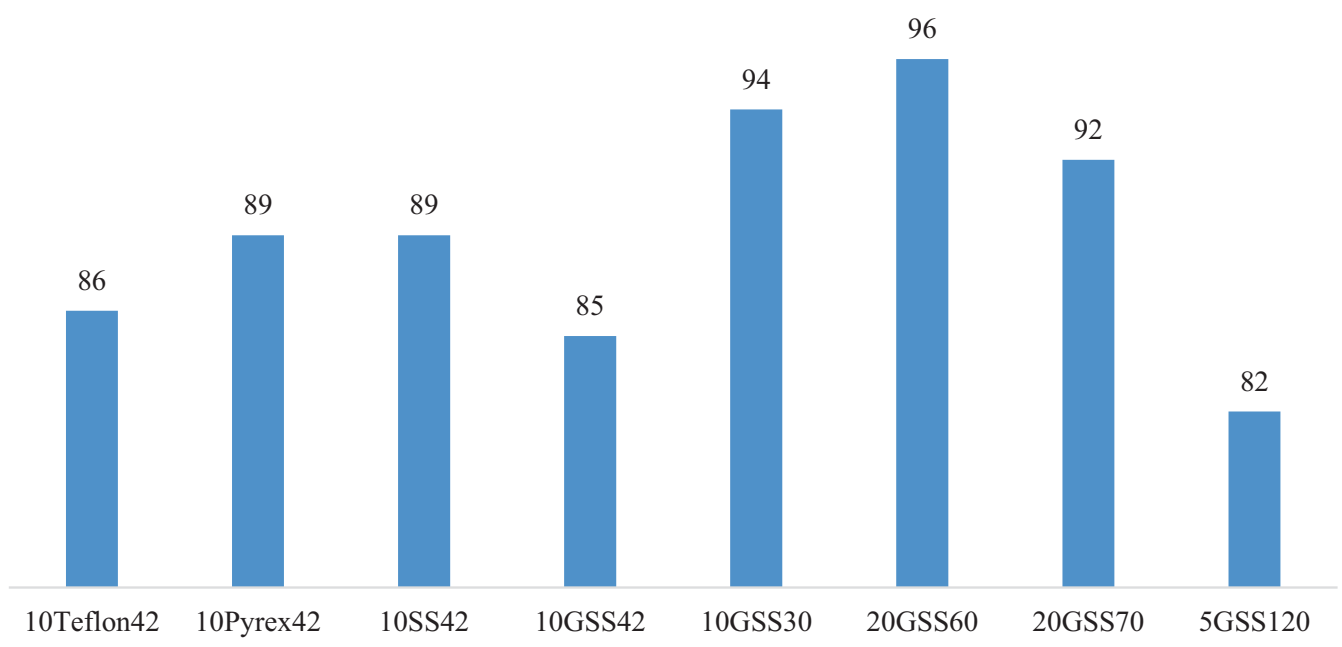

Figure 7. Calculated $\beta$ fraction of the PVDF films formed using different drying temperatures, different concentrations of PVDF and different substrates. 


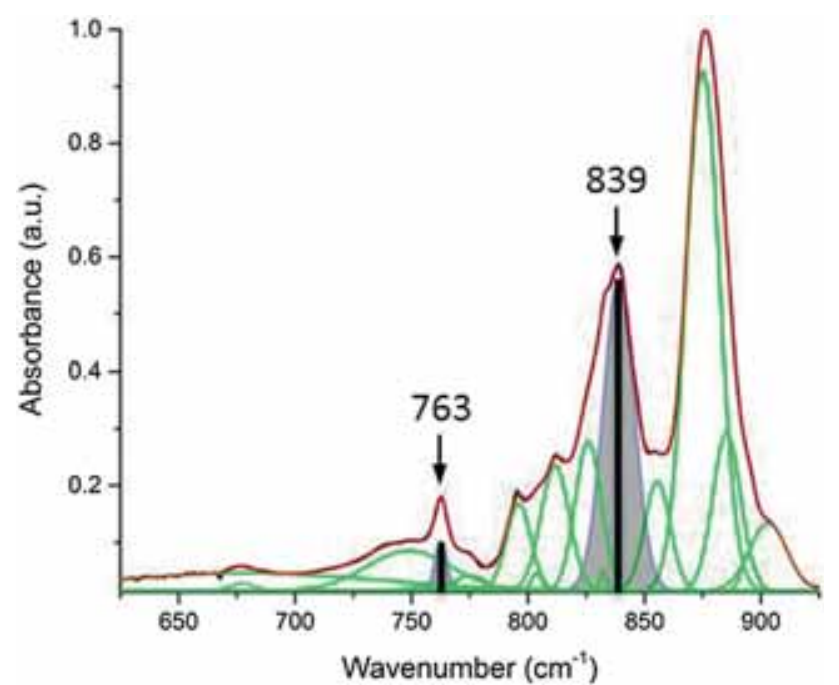

Figure 8. Deconvoluted FTIR spectrum of the PVDF film formed from $5 \mathrm{wt} \%$ of PVDF solution on a grounded stainless steel substrate and drying temperature of $120^{\circ} \mathrm{C}$. Black lines indicate the intensity of absorption bands at wavenumbers of 763 and $839 \mathrm{~cm}^{-1}$.

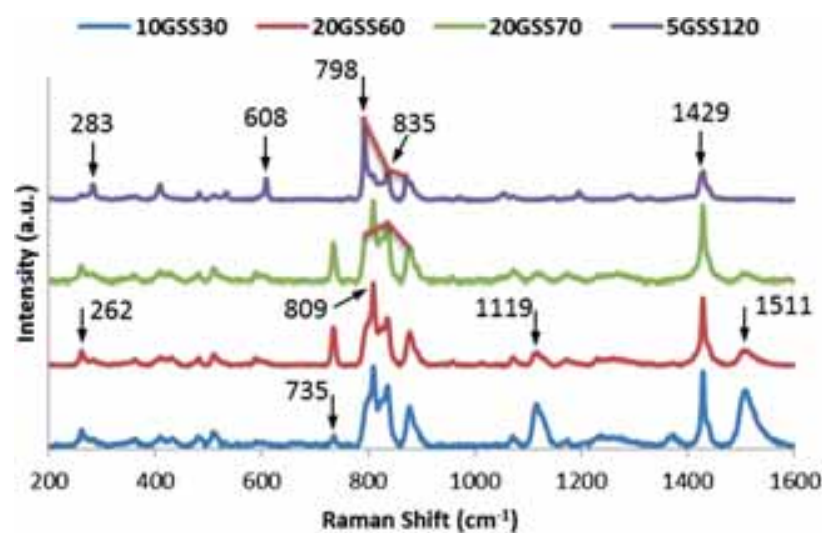

Figure 9. Recorded Raman spectroscopy of PVDF films formed using grounded stainless steel substrate, different PVDF concentrations and different drying temperatures. The Raman spectrum of all the films was recorded using excitation wavelength of $632.8 \mathrm{~nm}$ and power density of $10 \mathrm{~mW} \mathrm{~cm}^{-2}$.

using lower drying temperatures, it can be seen that the DMAc solvent-related Raman peaks at wavenumbers of 735,1119 and $1511 \mathrm{~cm}^{-1}$ are completely disappeared.

\section{Conclusion}

In this study, a series of PVDF films were systematically fabricated and studied using different concentrations of PVDF, different drying temperatures and different substrate materials. Effects of electrostatic field, substrate, solution concentration variation and different drying temperatures on degree of crystallinity, $\beta$-phase fraction and residual of solvent of the prepared PVDF films were also studied. The results show that electrostatic field reduces the solvent evaporation rate. Fabrication of a PVDF film with high crystallinity demands high drying temperature and substrate roughness, while the residual of the solvent and surface energy should be low. In general, $\beta$ fraction of fabricated PVDF film from PVDF/DMAc solution is high; however, it can be reduced by using higher concentration of PVDF and higher drying temperature.

\section{References}

[1] Martins P, Lopes A C and Lanceros-Mendez S 2014 Prog. Polym. Sci. 39683

[2] Bar-Cohen Y and Zhang Q 2008 MRS Bull. 33173

[3] Nalwa H S (ed) 1995 Ferroelectric polymers: chemistry: physics, and applications (CRC Press)

[4] Fukada E 2000 IEEE Trans. Ultrason, Ferroelectr. Freq. Control 471277

[5] Ueberschlag P 2001 Sens. Rev. 21118

[6] Chinaglia D L, Gregorio R, Stefanello J C, Pisani Altafim R A, Wirges W, Wang F et al 2010 J. Appl. Polym. Sci. 116785

[7] Salimi A and Yousefi A A 2003 Polym. Test. 22699

[8] Kepler R G and Anderson R A 1978 J. Appl. Phys. 494490

[9] Giannetti E 2001 Polym. Int. 5010

[10] Cardoso V F, Minas G, Costa C M, Tavares C J and LancerosMendez S 2011 Smart Mater. Struct. 20087002

[11] Gregorio R and Cestari M 1994 J. Polym. Sci. Part B: Polym. Phys. 32859

[12] Benz M and Euler W B 2003 J. Appl. Polym. Sci. 891093

[13] Salimi A and Yousefi A A 2004 J. Polym. Sci. Part B: Polym. Phys. 423487

[14] Gregorio R and Borges D S 2008 Polymer 494009

[15] Pramod K and Gangineni R B 2015 Bull. Mater. Sci. 381093

[16] Dhakras D, Borkar V, Ogale S and Jog J 2012 Nanoscale 4752

[17] Satapathy S, Pawar S, Gupta P K and Varma K B R 2011 Bull. Mater. Sci. 34727

[18] Tiwari V and Srivastava G 2014 J. Polym. Res. 211

[19] Aesar A, Poly(vinylidene fluoride), Product \# 44080, www. alfa.com

[20] Aesar A, N,N-Dimethylacetamide, 99\%, Product \# A10924, www.alfa.com

[21] Terasawa N and Asaka K 2014 Sens. Actuat. B: Chem. 193851

[22] Umebayashi Y, Matsumoto K, Watanabe M, Katoh K and Ishiguro S I 2001 Anal. Sci. 17323

[23] Ma W, Zhang J and Wang X 2008 Appl. Surf. Sci. 2542947

[24] De Gennes P G, Brochard-Wyart F and Quéré D 2013 Capillarity and wetting phenomena: drops, bubbles, pearls, waves (Springer Science \& Business Media)

[25] He L, Sun J, Wang X, Wang C, Song R and Hao Y 2013 Polym. Int. 62638

[26] Lin Y and Fan Y 2012 J. Appl. Polym. Sci. 125233

[27] Mohammadi B, Yousefi A A and Bellah S M 2007 Polym. Test. 2642

[28] Milani A, Castiglioni C and Radice S 2015 J. Phys. Chem. B 1194888

[29] Silva M P, Sencadas V, Botelho G, Machado A V, Rolo A G, Rocha J G et al 2010 Mater. Chem. Phys. 12287 